\title{
Electroweak Decays of Highly Charged lons
}

\author{
Ragandeep Singh Sidhu ${ }^{1,2, *}, J$. Glorius ${ }^{1}, Y u$. A. Litvinov ${ }^{1}, M$. K. Pavićević ${ }^{3}, M$. S. Sanjari ${ }^{1}$, \\ T. Stöhlker ${ }^{1,4,5}$, and the ILIMA, SPARC, LOREX, and NucAR Collaborations \\ ${ }^{1}$ GSI Helmholtzzentrum für Schwerionneforschung, 64291 Darmstadt, Germany \\ ${ }^{2}$ Physikalisches Institut, Ruprecht-Karls-Universität, 69120 Heidelberg, Germany \\ ${ }^{3}$ University of Salzburg, Department of Chemistry and Physics of Materials, 5020 Salzburg, Austria \\ ${ }^{4}$ Helmholtz-Institut Jena, 07743 Jena, Germany \\ ${ }^{5}$ Friedrich-Schiller-Universität Jena, 07743 Jena, Germany
}

\begin{abstract}
In this contribution we review the present status of experimental studies of electroweak decays of highly charged ions. A particular focus will be given on the bound state beta decay measurement of ${ }^{205} \mathrm{Tl}$.
\end{abstract}

\section{Introduction}

Investigations of decays of highly charged radionuclides (HCR) have been among the physics motivations for the construction of the Experimental Storage Ring (ESR) at GSI Helmholtz Center in Darmstadt, Germany [1]. The very reason for the interest in such studies is that HCRs with none or a few bound electrons, like, for instance, hydrogen-like (H-like), heliumlike (He-like) or lithium-like (Li-like) ions, represent well-defined nucleus plus lepton(s) systems with well-defined quantum numbers. Owing to the latter, HCRs offer unparalleled opportunities for studying the interplay of atomic structure and nuclear decay properties [2-9]. Apart from basic understanding of decay phenomena, such studies are essential for nuclear astrophysics where the nuclei involved into nucleosynthesis are highly ionized in hot and dense stellar environments [10-12].

The question on whether the nuclear decay probability is a fundamental constant or can be modified is addressed since the beginning of nuclear physics [13]. Only minor modifications of below about $1 \%$ have been observed by varying temperature, pressure, chemical environments, etc. [14-16]. However, it has been realized that nuclear half-lives $\left(T_{1 / 2}\right)$ can change dramatically in HCRs [17-19]. On the one hand, this is straightforward for fullyionized atoms where the decay branches involving atomic electrons, such as orbital electron capture (EC) or internal conversion (IC), are disabled. On the other hand, new decay channels disabled in neutral atoms can open up. One example of such exotic decay modes is the bound-state internal conversion (BIC) [20-22]. In the BIC process, an excited nuclear state resonantly transfers its excitation energy to a bound electron which is excited to a higher energy, but still bound, atomic level. This process has been discovered in single-pass experiments which are suitable for fast decays $\left(T_{1 / 2}<\sim 500 \mathrm{~ns}\right)$.

Since typical weak lifetimes are longer than about a ms, for their investigations one requires facilities which are capable to store the produced radionuclides in a preserved high charged state for a sufficiently long time. Apart from the recent studies in the Electron Beam

\footnotetext{
*e-mail: R.S.Sidhu@gsi.de
} 
Ion Trap (EBIT) at TRIUMF [23, 24], very recent measurements of weak decays of fullyionized ${ }^{49} \mathrm{Cr}^{24+},{ }^{53} \mathrm{Fe}^{26+}$ atoms [25] and the de-excitation of the ${ }^{94 m} \mathrm{Ru}^{44+}$ isomeric state [26] at the experimental cooler storage ring CSRe at Institute for Modern Physics (IMP), Lanzhou, all other investigations of decays of HCRs were performed in the ESR of GSI [2-4, 27].

\section{Heavy-lons Storage Rings}

There are three storage ring facilities in the world which allow for storing highly charged exotic nuclei [28]. These are the ESR at GSI [29], the CSRe at IMP [30] and the R3-storage ring at RIKEN Nishina Center in Wako [31-33].

Magnetic rigidity is defined $B \rho=m v \gamma / q$, where $m / q$ is mass to charge ratio of a particle, $v$ its velocity, and $\gamma$ relativistic Lorentz factor. The high energy part of the GSI facility consists of an 18-Tm heavy-ion synchrotron SIS, the in-flight projectile fragment separator FRS [34] and the 10-Tm cooler-storage ring ESR [35]. Exotic nuclei are produced at relativistic energies of a few hundreds $\mathrm{MeV} / \mathrm{u}$ through projectile fragmentation or in-flight fission nuclear reactions [36]. The FRS is employed to select and transport the ions of interest to the ESR [37]. The flight time through the FRS is merely 300 ns. In general the FRS can transport all produced exotic nuclei, the "cocktail" beam, lying within its $B \rho$ acceptance. By adding specially shaped degraders, one employs the atomic energy loss, $\Delta E$, in the so-called $B \rho-\Delta E-B \rho$ separation method to achieve the separation of mono-isotopic beams [38].

Due to the high kinetic energies, orbital electrons are efficiently stripped off the fragments emerging from the target material [39-42]. By tuning the projectile energy, target material and thickness, ion beams in a specific charge state of interest can selectively be produced and stored in the ESR. Fully-ionised and up to 4-electron ions are routinely produced at energies of about $100-400 \mathrm{MeV} / \mathrm{u}$, see, e.g., Refs. [43-54].

The storage ring mass spectrometry makes use of cocktail beams and is applied successfully at the ESR [56-67] and CSRe [68-77]. The R3 is being commissioned now and is expected to begin with mass measurements already in 2018.

\section{Decay studies of highly charged radionuclides}

The decay studies are typically conducted with mono-isotopic beams to avoid contaminations or secondary feedings. Furthermore, mono-isotopic beams are often needed in reaction studies with internal targets or electron cooler (see, e.g., [43, 78-80]).

The ESR has a circumference of $108 \mathrm{~m}$. This is an ultra-high vacuum machine with an average residual gas pressure of about $10^{-10}-10^{-11}$ mbar. The latter is an essential prerequisite for experiments with highly charged ions. The beam cooling is needed to reduce the inevitable momentum spread of the ions, $\Delta p / p$, which is mainly due to nuclear reaction process. This is of utmost importance to achieve sufficient resolving power for an unambiguous identification of parent and daughter ions. Stochastic [81] and electron [82] cooling methods are used to reduce $\Delta p / p$ to $\approx 10^{-5}-10^{-7}$ within a few seconds. The achievable minimum $\Delta p / p$ depends critically on the number of stored ions and is determined by the balance between the cooling force and the intra-beam scattering effects. For electron-cooled ions, a transition to ordered beams is observed at low particle intensities of below about 2000 ions [83, 84]. In this mode, the mass resolving power of about 750000 is reached, which is sufficient to separate isobars and even low-lying isomers by their revolution frequencies in the ring. The intensities of stored ions are continuously monitored with non-destructive time-resolved Schottky spectrometry [85-89]. In addition, the decay/reaction products can be intercepted by dedicated particle detectors [90-93]. 
Numerous experiments have been performed at the ESR and since recently also at the CSRe for investigations of continuum beta decay and de-excitation of nuclear isomers [25, 26, 94-103]. In the last years a special attention is given to two-body beta decays. These are the orbital electron capture (EC) and bound-state $\beta^{-}$-decay $\left(\beta_{b}^{-}\right)$, which can be described in a general form $n+v_{e} \leftrightarrow p+e_{b}^{-}$, where $p, n, e_{b}^{-}, v_{e}$ are proton, neutron, bound electron and electron neutrino, respectively. Concerning the experiments on EC, the reader is referred to Refs. [104-121]. Here we focus on the $\beta_{b}^{-}$decay studies.

In the $\beta_{b}^{-}$, one of the neutrons in the nucleus is transmuted into a proton with an emission of an electron and an electron antineutrino. However, different from an ordinary $\beta_{c}^{-}$decay, the electron is not emitted to the continuum but occupies one of the bound orbitals [17]. This decay was first predicted by Daudel et al. . [122] in 1947 and then discussed in detail by Bahcall [123]. Similar to EC, in which the electron from any shell can be captured, the created electron in the $\beta_{b}^{-}$-decay can occupy different shells in a daughter ion. The scaling of the probabilities to capture (generate) s-electron from (in) electron shells with different principal quantum number $n$ scales as $1 / n^{3}$ [124]. Since the inner orbitals in neutral atoms are Pauli-blocked, $\beta_{b}^{-}$is only a marginal decay branch in neutral atoms.

The first direct observation of the bound-state beta decay ( $\beta_{b}$ decay) was done in the ESR in 1992 [125] with the use of bare ${ }^{163} \mathrm{Dy}^{66+}$ ions. Neutral ${ }^{163}$ Dy is stable. However, without electrons its half-life is merely $\sim 50$ days. This result yielded the temperature $T$ for the branching point of the $s$-process at $A=163$ [2]. Another example is ${ }^{187} \mathrm{Re}$ atom, which has a very long half-life of $42 \mathrm{~Gy}$. However, the increased $Q$-value in ${ }^{187} \mathrm{Re}^{75+}$ ions enables the decay to the first exited state in ${ }^{187} \mathrm{Os}$. The measured $T_{1 / 2}$ is merely 33 years [126], which constrains the applicability of the ${ }^{187} \mathrm{Re} /{ }^{187} \mathrm{Os}$ pair in cosmo-chronometry [127].

Fully-ionised ${ }^{205} \mathrm{Hg}^{80+}$ and ${ }^{206,207} \mathrm{Tl}^{81+}$ nuclei have sufficiently large decay $Q$-values. The parent and daughter ions could thus be directly resolved by their revolution frequencies in the ESR. Both $\beta_{b}^{-}$- and $\beta_{c}^{-}$-decay branches were measured $[128,129]$. In contrast to numerous measurements of $\mathrm{EC} / \beta_{c}^{+}$branching ratios, these are the only measured $\beta_{b}^{-} / \beta_{c}^{-}$ratios, which are in fair agreement with theoretical estimations [18, 129].

\section{Bound-State Beta Decay of ${ }^{205} \mathrm{TI}^{81+}$}

The measurement of the bound-state beta-decay rate of fully-ionized ${ }^{205} \mathrm{Tl}$ has been proposed more than 20 years ago $[130,131]$. Its decay rate is needed to determine the matrix element for the EC of the $2.3 \mathrm{keV}$ excited state in ${ }^{205} \mathrm{~Pb}$ to the ground state of ${ }^{205} \mathrm{Tl}$. This matrix element is important for the determination of the pp-solar neutrino capture probability into the $2.3 \mathrm{keV}$ state of ${ }^{205} \mathrm{~Pb}[132,133]$. It can also be used to constrain the very end of the s-process nucleosynthesis [134-137]. The proposal for this measurement has been approved at GSI.

A projectile beam of enriched ${ }^{206} \mathrm{~Pb}$ will be accelerated by SIS to $\sim 700 \mathrm{MeV} / \mathrm{u}$. The intensity of $2 \cdot 10^{9}$ particles/spill on target is expected. The production target will be ${ }^{9} \mathrm{Be}$. The masses of parent ${ }^{205} \mathrm{Tl}^{81+}$ and daughter ${ }^{205} \mathrm{~Pb}^{81+}$ ions differ by $\sim 31 \mathrm{keV}$. This tiny difference does not allow to separate them by revolution frequencies in the ESR. Therefore, the essential condition for this experiment is that the amount of ${ }^{205} \mathrm{~Pb}^{81+}$ ions directly injected into the ESR is as small as possible. For this purpose we will employ the $B \rho-\Delta E-B \rho$ separation. The thicknesses of all matter in the FRS will be optimized such that the final energy of ${ }^{205} \mathrm{Tl}^{81+}$ ions equals $400 \mathrm{MeV} / \mathrm{u}$. The ESR stochastic cooling system is matched to this energy. Beam stacking will be applied to accumulate about $10^{6}$ parent ions.

In order to count the number of produced $\beta_{b}^{-}$-daughters, the bound electron will be stripped from ${ }^{205} \mathrm{~Pb}$ by employing a strong Ar gas-jet. The gas jet has a density of about $10^{13}$ argon atoms $/ \mathrm{cm}^{2}$. It will be turned-on for a fixed period of time. The change of the 
atomic charge state causes a significant alteration in the trajectory and hence the revolution frequency. The number of daughter ions can be counted in two ways either by Schottky diagnosis or by a particle detector on the corresponding orbit.

For the design number of $10^{6}$ stored bare ${ }^{205} \mathrm{Tl}$ ions and a very cautiously estimated bound-state beta decay half-life of 1 year, we expect about 40 bound-state beta decays within a storage time of 1 hour. A waiting time is therefore necessary to accumulate daughter ions before the start of the gas-jet. We plan to have several waiting times of up to several hours. During the waiting time, the electron current in the cooler will be set to the minimum to reduce the recombination rate. The intensity of the parent ions will constantly be monitored by the Schottky analysis. This is essential to account for various beam losses.

The amount of the ${ }^{205} \mathrm{~Pb}^{81+}$ ions directly injected from the FRS will be measured by switching the gas-jet immediately after injection. The primary beam of ${ }^{206} \mathrm{~Pb}$ in charge states $82+$ and $81+$ will be used to calibrate the efficiency of the gas-jet stripping and beam losses.

\section{Outlook}

Heavy-ion storage-cooler rings have proven to be excellent tools for performing highprecision decay experiments on HCRs. There are many ideas for unique investigations of decays of HCRs which guarantee the rich perspectives of the field, see [112, 116, 138-142].

New storage ring projects are launched worldwide. The CRYRING has been installed behind the ESR [143], where HCRs decelerated to energies down to a few hundreds of A $\mathrm{keV}$ will be available. This will enable unique experiments at the interface between nuclear structure, atomic and astrophysics. Since the TSR@ISOLDE project at CERN [144] has been postponed, the TSR will probably be installed at IMP. Decays of HCRs belong to major physics cases for the TSR [144]. New storage rings are in the focus of the next-generation radioactive-ion beam facilities FAIR in Germany and HIAF in China. At FAIR, the stored HCRs will be available at energies covering 10 orders of magnitude from nearly at rest to about 5 A GeV [145-147]. Some discussions on the research with HCRs at FAIR and HIAF can be found in [148, 149].

This short paper is dedicated to our friends Paul Kienle and Fritz Bosch who made indispensable contributions to this field of research. This review is based on previous publications of our colleagues from several storage ring collaborations. To all of them we are deeply obliged. This work is in part supported by the Helmholtz/CAS Joint Research Group (HCJRG-108), and by the European Research Council (ERC) under the EU Horizon 2020 research and innovation programme (ERC CG 682841 "ASTRUm”).

\section{References}

[1] P. Kienle Forschung im Focus (Interfrom, 1993).

[2] Yu. A. Litvinov and F. Bosch, Rep. Prog. Phys. 74, 016301 (2011).

[3] Yu. A. Litvinov et al., Phys. Scripta T 144, 014001 (2011).

[4] F. Bosch et al., Prog. Part. Nucl. Phys. 73, 8 (2013).

[5] F. Bosch et al., Phys. Scripta T 156, 014025 (2013).

[6] D. R. Atanasov et al., Phys. Scripta T 156, 014026 (2013).

[7] F. Bosch and Yu. A. Litvinov, Int. J. Mass Spectrom. 349-350, 151 (2013).

[8] D. R. Atanasov et al., J. Phys. B 48, 144024 (2015).

[9] D. R. Atanasov et al., J. Phys. Conf. Series 875, 012008 (2017).

[10] E. M. Burbidge et al., Rev. Mod. Phys. 29, 547 (1957).

[11] H. Schatz, Int. J. Mass Spectrom. 349-350, 181 (2013). 
[12] K. Langanke and G. Pinedo, Phys. Scripta T 166, 014001 (2015).

[13] E. Rutherford and F. Soddy, J. Chem. Soc. Trans. 81, 837 (1902).

[14] G. T. Emery, Ann. Rev. Nucl. Sci. 22, 165 (1972).

[15] B. Wang et al., Eur. Phys. J. A 28, 375 (2006).

[16] T. Ohtsuki et al., Phys. Rev. Lett. 98, 252501 (2004).

[17] J. N. Bahcall, Phys. Rev. 126, 1143 (1962).

[18] K. Takahashi and K. Yokoi, Nucl. Phys. A 404, 578 (1983).

[19] K. Takahashi and K. Yokoi, At. Data Nucl. Data Tables 36, 375 (1987).

[20] W. R. Phillips et al., Phys. Rev. Lett. 62, 1025 (1989).

[21] W. R. Phillips et al., Phys. Rev. A 473682 (1993).

[22] F. Attallah et al., Phys. Rev. C 55, 1665 (1997).

[23] K. G. Leach et al., Nucl. Instr. Meth. A 780, 91 (2015).

[24] K. G. Leach et al., Atoms 5, 14 (2017).

[25] X. L. Tu et al., Phys. Rev. C 97, 014321 (2018).

[26] Q. Zeng et al., Phys. Rev. C 96, 031303(R) (2017).

[27] D. Atanasov et al., J. Phys. Conf. Series 875, 012008 (2017).

[28] Y. H. Zhang et al., Phys. Scripta 91, 073002 (2016).

[29] B. Franzke, H. Geissel and G. Münzenberg, Mass Spectrometry Reviews 27, 428 (2008).

[30] J. W. Xia et al., Nucl. Instr. Meth. A 488, 11 (2002).

[31] Y. Yamaguchi et al., Nucl. Instr. Meth. B 266, 4575 (2008).

[32] A. Ozawa et al., Prog. Theor. Exper. Phys. 2012, 03 C009 (2012).

[33] T. Yamaguchi et al., Int. J. Mass Spectrom. 349-350, 240 (2013).

[34] H. Geissel et al., Nucl. Instr. Meth. B 70, 286 (1992).

[35] B. Franzke, Nucl. Instr. Meth. B 24/25, 18 (1987).

[36] H. Geissel, Progress Part. Nucl. Phys. 42, 3 (1999).

[37] H. Geissel et al., Phys. Rev. Lett. 68, 3412 (1992).

[38] G. Münzenberg, Nucl. Instr. Meth. B 70, 265 (1992).

[39] C. Scheidenberger et al., Nucl. Instr. Meth. B 142, 441 (1998).

[40] H. Ogawa et al., Phys. Rev. A 75, 020703(R) (2007).

[41] I. Tolstikhina et al., Phys. Usp. 56, 213 (2013). [2013 Usp. Fiz. Nauk 183 225]

[42] V. P. Shevelko et al., Nucl. Instr. Meth. B 421, 45 (2018).

[43] C. Brandau et al., J. Phys. Conf. Series 194, 012023 (2009).

[44] C. Brandau et al., J. Phys. Conf. Series 388, 062042 (2012).

[45] W. Nörtershäuser et al., Phys. Scripta T 156, 014016 (2013).

[46] C. Brandau et al., Phys. Scripta T 156, 014050 (2013).

[47] S. Tashenov et al., Phys. Rev. Lett. 113, 113001 (2014).

[48] M. Lochmann et al., Phys. Rev. A 90 030501(R) (2014).

[49] P-M Hillenbrand et al., Phys. Rev. A 91, 022705 (2015).

[50] P-M Hillenbrand et al., Phys. Rev. A 93, 042709 (2016).

[51] H. Beyer et al., J. Phys. B 48, 144010 (2015).

[52] J. Ullmann et al., J. Phys. B 48, 144022 (2015).

[53] R. Sanchez et al., J. Phys. B 50, 085004 (2017).

[54] J. Ullmann et al., Nature Comm. 8, 15484 (2017).

[55] A. Gumberidze et al., Nucl. Instr. Meth. B 408, 27 (2017).

[56] T. Radon et al., Nucl. Phys. A 677, 75 (2000).

[57] Yu. N. Novikov et al., Nucl. Phys. A 697, 92 (2002). 
[58] J. Stadlmann et al., Phys. Lett. B 586, 27 (2004).

[59] Yu. A. Litvinov et al., Phys. Rev. Lett. 95, 042501 (2005).

[60] H. Geissel and Yu. A. Litvinov, J. Phys. G 31, S1779 (2005).

[61] Yu. A. Litvinov et al., Nucl. Phys. A 756, 3 (2005).

[62] H. Geissel et al., Eur. Phys. J Special Topics 150, 109 (2007).

[63] Yu. A. Litvinov et al., Nucl. Phys. A 787, 315c (2007).

[64] B. H. Sun et al., Nucl. Phys. A 812, 1 (2008).

[65] B. H. Sun et al., Int. J. Mod. Phys E 18346 (2009).

[66] L. X. Chen et al., Phys. Rev. Lett. 102, 122503 (2009).

[67] D. Shubina et al., Phys. Rev. C 88, 024310 (2013).

[68] M. Wang et al., Int. J. Mod. Phys. E 18, 352 (2009).

[69] X. L. Tu et al., Nucl. Instr. Meth. A 654, 213 (2011).

[70] X. L. Tu et al., Phys. Rev. Lett. 106, 112501 (2011).

[71] Y. H. Zhang, Phys. Rev. Lett. 109, 102501 (2012).

[72] H. S. Xu et al., Int. J. Mass. Spectr. 349-350, 162 (2013).

[73] X. L. Yan et al., Astrophys. J. Lett. 766, L8 (2013).

[74] X. L. Tu et al., J. Phys. G 41, 025104 (2014).

[75] P. Shuai et al., Phys. Lett. B 735, 327 (2014).

[76] X. Xu et al., Phys. Rev. Lett. 117, 182503 (2016).

[77] P. Zhang et al., Phys. Lett. B 767, 20 (2017).

[78] N. Kalantar-Nayestanaki et al., Int. J. Mod. Phys. E 18, 524 (2009).

[79] B. Mei et al., Phys. Rev. C 92, 035803 (2015).

[80] H. Moeini et al., Nucl. Instr. Meth. A 634, 77 (2011).

[81] F. Nolden et al., Nucl. Instr. Meth. A 532, 329 (2004).

[82] M. Steck et al., Nucl. Instr. Meth. A 532, 357 (2004).

[83] M. Steck et al., Phys. Rev. Lett. 77, 3803 (1996).

[84] J. P. Schiffer and P. Kienle, Z. Phys. A 321, 181 (1985).

[85] Yu. A. Litvinov et al., Nucl. Phys. A 734, 473 (2004).

[86] H. Geissel et al., Nucl. Phys. A 746, 150c (2004).

[87] H. Geissel et al., Eur. Phys. J. Special Topics 150, 109 (2007).

[88] F. Nolden et al., Nucl. Instr. Meth. A 659, 69 (2011).

[89] X. C. Chen et al., Nucl. Instr. Meth. A 826, 39 (2016).

[90] M. A. Najafi et al., Nucl. Instr. Meth. A 836, 1 (2016).

[91] B. Mei et al., Phys. Rev. C 94, 044615 (2016).

[92] O. Klepper et al., Nucl. Instr. Meth. B 70, 427 (1992).

[93] O. Klepper and C. Kozhuharov, Nucl. Instr. Meth. B 204, 553 (2003).

[94] H. Irnich et al., Phys. Rev. Lett. 75, 4182 (1995).

[95] F. Attallah et al., Nucl. Phys. A 701, 561c (2002).

[96] Yu. A. Litvinov et al., Phys. Lett. B 573, 80 (2003).

[97] B. H. Sun et al., Eur. Phys. J. A 31, 393 (2007).

[98] B. H. Sun et al., Phys. Lett. B 688, 294 (2010).

[99] M. W. Reed et al., Phys. Rev. Lett. 105, 172501 (2010).

[100] L. X. Chen et al., Phys. Lett. B 691, 234 (2010).

[101] M. W. Reed et al., Phys. Rev. C 86, 054321 (2012).

[102] L. X. Chen et al., Phys. Rev. Lett. 110, 122502 (2013).

[103] A. Akber et al., Phys. Rev. C 91, 031301(R) (2015). 
[104] L. M. Folan and V. I. Tsifrinovich, Phys. Rev. Lett. 74, 499 (1995).

[105] Yu. A. Litvinov et al., Phys. Rev. Lett. 99, 262501 (2007).

[106] Z. Patyk et al., Phys. Rev. C 77, 014306 (2008).

[107] Yu. A. Litvinov, Nucl. Phys. A 805, 260c (2008).

[108] Yu. A. Litvinov et al., Phys. Lett. B 664, 162 (2008).

[109] P. A. Vetter et al., Phys. Lett. B 670, 196 (2008).

[110] A. N. Ivanov et al., Phys. Rev. C 78, 025503 (2008).

[111] T. Faestermann et al., Phys. Lett. B 672, 227 (2009).

[112] Yu. A. Litvinov, Int. J. Mod. Phys. E 18, 323 (2009).

[113] N. Winckler et al., Phys. Lett. B 679, 36 (2009).

[114] C. Giunti, Nucl. Phys. B (Proc. Suppl.) 188, 43 (2009).

[115] A. Merle, Phys. Rev. C 80, 054616 (2009).

[116] N. Winckler et al., Nucl. Phys. A 834, 432c (2010).

[117] F. Bosch and Yu. A. Litvinov, Prog. Part. Nucl. Phys. 64, 435 (2010).

[118] K. Siegien-Iwaniuk et al., Phys. Rev. C 84, 014301 (2011).

[119] D. R. Atanasov et al., Eur. Phys. J. A 48, 22 (2012).

[120] P. Kienle et al., Phys. Lett. B 726, 638 (2013).

[121] A. Gal, Symmetry 8, 49 (2016).

[122] R. Daudel et al., J. Phys. Radium 8, 238 (1948).

[123] J. N. Bahcall, Phys. Rev. 124, 495 (1961).

[124] W. Bambynek et al., Rev. Mod. Phys. 49, 77 (1977).

[125] M. Jung et al., Phys. Rev. Lett. 69, 2164 (1992).

[126] F. Bosch et al., Phys. Rev. Lett. 77, 5190 (1996).

[127] F. Bosch et al., AIP Conf. Proc. 477, 344 (1999).

[128] T. Ohtsubo et al., Phys. Rev. Lett. 95, 052501 (2005).

[129] J. Kurcewicz et al., Acta Phys. Polonica B 41, 525 (2010).

[130] W. F. Henning et al., AIP Conf. Proc. 126, 203 91985).

[131] M. K. Pavićević, Nucl. Instr. Meth. A 271, 287 (1988).

[132] M. K. Pavićević et al., Nucl. Instr. Meth. A 621, 282 (2010).

[133] M. K. Pavićević et al., J. Phys. Conf. Series 888, 012192 (2017).

[134] J. B. Blake, T. Lee and D. N. Schramm, Nature Phys. Sci. 242, 98 (1973).

[135] J. B. Blake and D. N. Schramm, Astroph. J. 197, 615 (1975).

[136] G. Huss et al., Geoch. Cosmoch. Acta 73, 4922 (2009).

[137] R. Baker et al., Earth and Planet. Sci. Lett. 291, 39 (2010).

[138] C. Scheidenberger et al., Hyperf. Interact. 173, 61 (2006).

[139] F. Bosch et al., EPJ Web Conf. 123, 04003 (2016).

[140] C. Nociforo et al., Phys. Scripta T 150, 014028 (2012).

[141] Ch. J. Cerjan et al., J. Phys. G 45, 033003 (2018).

[142] I. Dillmann and Yu. A. Litvinov, Prog. Part. Nucl. Phys. 66, 358 (2011).

[143] M. Lestinsky et al., Eur. Phys. J. Special Topics 225, 797 (2016).

[144] M. Grieser et al., Eur. Phys. J. Special Topics 207, 1 (2012).

[145] P. M. Walker et al., Int. J. Mass Spectrom. 349-350, 247 (2013).

[146] T. Stöhlker et al., Hyperf. Interact. 227, 45 (2014).

[147] T. Stöhlker et al., Nucl. Instr. Meth. B 365, 680 (2015).

[148] Yu. A. Litvinov et al., Nucl. Instr. Meth. B 317, 603 (2013).

[149] P. J. Woods et al., Phys. Scripta T 166, 014002 (2015) 